\title{
ECNU: Expression- and Message-level Sentiment Orientation Classification in Twitter Using Multiple Effective Features
}

\author{
Jiang Zhao ${ }^{\dagger}$, Man Lan*, Tian Tian Zhu ${ }^{\dagger}$ \\ Department of Computer Science and Technology \\ East China Normal University \\ †51121201042,51111201046@ecnu.cn; *mlan@cs.ecnu.edu.cn
}

\begin{abstract}
Microblogging websites (such as Twitter, Facebook) are rich sources of data for opinion mining and sentiment analysis. In this paper, we describe our approaches used for sentiment analysis in twitter (task 9) organized in SemEval 2014. This task tries to determine whether the sentiment orientations conveyed by the whole tweets or pieces of tweets are positive, negative or neutral. To solve this problem, we extracted several simple and basic features considering the following aspects: surface text, syntax, sentiment score and twitter characteristic. Then we exploited these features to build a classifier using SVM algorithm. Despite the simplicity of features, our systems rank above the average.
\end{abstract}

\section{Introduction}

Microblogging services such as Twitter $^{1}$, Facebook $^{2}$ today play an important role in expressing opinions on a variety of topics, discussing current issues or sharing one's feelings about different objects in our daily life (Agarwal and Sabharwal, 2012). Therefore, Twitter (and other platforms) has become a valuable source of users' sentiments and opinions and with the continuous and rapid growth of the number of tweets, analyzing the sentiments expressed in twitter has attracted more and more researchers and communities, for example, the sentiment analysis task in twitter was held in

\footnotetext{
This work is licensed under a Creative Commons Attribution 4.0 International Licence. Page numbers and proceedings footer are added by the organisers. Licence details: http://creativecommons.org/licenses/by/4.0/

${ }^{1}$ http://twitter.com

${ }^{2}$ http://facebook.com/
}

SemEval 2013 (Nakov et al., 2013). It will benefit lots of real applications such as simultaneously businesses, media outlets, and help investors to discover product trends, identify customer preferences and categorize users by analyzing these tweets (Becker et al., 2013).

The task of sentiment analysis in twitter in SemEval 2014 (Sara et al., 2014) aims to classify whether a tweet's sentiment is positive, negative or neutral at expression level or message level. The expression-level subtask (i.e., subtask A) is to determine the sentiment of a marked instance of a word or phrase in the context of a given message, while the message-level subtask (i.e., subtask B) aims to determine the sentiment of a whole message. Previous work (Nakov et al., 2013) showed that message-level sentiment classification is more difficult than that of expression-level (i.e., $0.690 \mathrm{vs}$ 0.889 in terms of $F$-measure) since a message may be composed of inconsistent sentiments.

To date, lots of approaches have been proposed for conventional blogging sentiment analysis and a very broad overview is presented in (Pang and Lee, 2008). Inspired by that, many features used in microblogging mining are adopted from traditional blogging sentiment analysis task. For example, $n$-grams at the character or word level, part-of-speech tags, negations, sentiment lexicons were used in most of current work (Agarwal et al., 2011; Barbosa and Feng, 2010; Zhu et al., 2013; Mohammad et al., 2013; Kökciyan et al., 2013). They found that $n$-grams are still effective in spite of the short length nature of microblogging and the distributions of different POS tags in tweets with different polarities are highly different (Pak and Paroubek, 2010). Compared with formal blog texts, tweets often contain many informal writings including slangs, emoticons, cre- 
ative spellings, abbreviations and special marks (i.e., mentions @ and hashtags \#), and thus many twitter-specific features are proposed to characterize this phenomena. For example, features record the number of emoticons, elongated words and hashtags were used in (Mohammad et al., 2013; Zhu et al., 2013; Kökciyan et al., 2013). In this work, we adopted many features from previous work and then these features were fed to SVM to perform classification.

The remainder of this paper is organized as follows. Section 2 describes our systems including preprocessing, feature representations, data sets, etc. Results of two subtasks and discussions are reported in Section 3. Finally, we conclude this paper in Section 4.

\section{Our Systems}

We extracted eight types of features and the first six types were used in subtask $A$ and all features were used in subtask B. Then, several classification algorithms were examined on the development data set and the algorithm with the best performance was chosen in our final submitted systems.

\subsection{Preprocessing}

In order to remedy as many informal texts as possible, we recovered the elongated words to their normal forms, e.g., "goooooood" to "good" and collected about five thousand slangs or abbreviations from Internet to convert each slang to its complete form, e.g., "Idering" to "wondering", " $2 g 2 b 4 g "$ to "to good to be forgotten". Then these preprocessed texts were used to extract non twitter-specific features (i.e., POS, lexicon, $n$ grams, word cluster and indicator feature).

\subsection{Feature Representations}

\subsubsection{POS Features}

(Pak and Paroubek, 2010) found that POS tags help to identify the sentiments of tweets and they pointed out that objective tweets often contain more nouns than subjective tweets and subjective tweets may carry more adjectives and adverbs than objective tweets. Therefore, we used Stanford POS Tagger ${ }^{3}$ and recorded the number of four different tags for each tweet: noun (the corresponding POS tags are "NN", "NNP", "NNS" and "NNPS"), verb (the corresponding POS tags

\footnotetext{
${ }^{3}$ http://nlp.stanford.edu/software/tagger.shtml
}

are "VB", "VBD", "VBG", "VBN", "VBP" and "VBZ"), adjective (the corresponding POS tags are "JJ", "JJR" and "JJS") and adverb (the corresponding POS tags are "RB", "RBR" and "RBS"). Then we normalized them by the length of given instance or message.

\subsubsection{Sentiment Lexicon-based Features}

Sentiment lexicons are widely used to calculate the sentiment scores of phrases or messages in previous work (Nakov et al., 2013; Mohammad et al., 2013) and they are proved to be very helpful to detect the sentiment orientation. Given a phrase or message, we calculated the following six feature values: (1) the ratio of positive words to all words, i.e., the number of positive words divided by the number of total words; (2) the ratio of negative words to all words; (3) the ratio of objective words to all words; (4) the ratio of positive sentiment score to the total score (i.e., the sum of the positive and negative score); (5) the ratio of negative sentiment score to the total score; (6) the ratio of positive score to negative score, if the negative score is zero, which means this phrase or message has a very strong positive sentiment orientation, we set ten times of positive score as its value.

During the calculation, we also considered the effects of negation words since they may reverse the sentiment orientation in most cases. To do so, we defined the negation context as a snippet of a tweet that starts with a negation word and ends with punctuation marks. If a non-negation word is in a negation context and also in the sentiment lexicon, we reverse its polarity. For example, the word "bad" in phrase "not bad" originally has a negative score of 0.625 , after reversal, this phrase has a positive score of 0.625 . A manually made list containing 29 negation words (e.g., no, hardly, never, etc) was used in our experiment.

Four sentiment lexicons were used to decide whether a word is subjective or objective and obtain its sentiment score.

MPQA (Wilson et al., 2009). This subjectivity lexicon contains about 8000 subjective words and each word has two types of sentiment strength: strong subjective and weak subjective, and four kinds of sentiment polarities: positive, negative, both (positive and negative) and neutral. We used this lexicon to determine whether a word is positive, negative or objective and assign a value of 0.5 or 1 if it is weak or strong subjective (i.e., positive or negative) respectively. 
SentiWordNet(SWN) (Baccianella et al., 2010). This sentiment lexicon contains about 117 thousand items and each item corresponds to a synset of WordNet. Three sentiment scores: positivity, negativity, objectivity are provided and the sum of these three scores is always 1 , for example, living\#a\#3, positivity: 0.5, negativity: 0.125 , objectivity: 0.375 . In experiment we used the most common sense of a word.

NRC (Mohammad et al., 2013). Mohammad et al. collected two sets of tweets and each tweet contains the seed hashtags or emoticons and then they labeled the sentiment orientation for each tweet according to its hashtags or emoticons. They used pointwise mutual information (PMI) to calculate the sentiment score for each word and obtained two sentiment lexicons (i.e., hashtag lexicon and emoticon lexicon).

IMDB. We generated an unigram lexicon by ourselves from a large movie review data set from IMDB website (Maas et al., 2011) which contains 25,000 positive and 25,000 negative movie reviews by calculating their PMI scores.

\subsubsection{Word $n$-Gram}

Words in themselves in tweets usually carry out the original sentiment orientation, so we consider word $n$-grams as one feature. We removed URLs, mentions, hashtags, stopwords from tweet and then all words were stemmed using the nltk ${ }^{4}$ toolkit. For subtask A, only unigram was used and we used word frequency as feature values. For subtask $\mathrm{B}$, both unigram and bigram were used. Besides, weighted unigram was also used where we replaced word frequency with their sentiment scores using the hashtag lexicon and emoticon lexicon in NRC.

\subsubsection{Twitter-specific Features}

Punctuation Generally, punctuation may express users' sentiment in a certain extent. Therefore we recorded the frequency of the following four types of punctuation: exclamation (!), question (?), double (") and single marks ('). In addition, we also recorded the number of contiguous sequences of exclamation marks, question marks, and both of them which appeared at the end of a phrase or message.

Emoticon Emoticons are widely used to directly express the sentiment of users and thus we counted

\footnotetext{
${ }^{4}$ http://nltk.org/
}

the number of positive emoticons, negative emoticons and the sum of positive and negative emoticons. To identify the polarities of emoticons, we collected 36 positive emoticons and 33 negative emoticons from the Internet.

Hashtag A hashtag is a short phrase that concatenates more than one words together without white spaces and users usually use hashtags to label the subject topic of a tweet, e.g., \#toobad, \#ihateschool, \#NewGlee. Since a hashtag may contain a strong sentiment orientation, we first used the Viterbi algorithm (Berardi et al., 2011) to split hashtags and then calculated the sentiment scores of hashtags using the hashtag and emoticon lexicon in NRC.

\subsubsection{Word Cluster}

Apart from $n$-gram, we presented another word representations based on word clusters to explore shallow semantic meanings and reduced the sparsity of the word space. 1000 word clusters provided by CMU pos-tagging tool ${ }^{5}$ were used to represent tweet contents. For each tweet we recorded the number of words from each cluster, resulting in 1000 features.

\subsubsection{Indicator Features}

We observed that the polarity of a message sometimes is revealed by some special individual positive or negative words in a certain degree. However the sentiment lexicon based features where a synthetical sentiment score of a message is calculated may hide this information. Therefore, we directly used several individual sentiment scores as features. Specifically, we created the following sixteen features for each message where the hashtag and emoticon lexicons were used to obtain sentiment scores: the sentiment scores of the first and last sentiment-bearing words, the three highest and lowest sentiment scores.

\subsection{Data sets and Evaluation Metric}

The organizers provide tweet ids and a script for all participants to collect data. Table 1 shows the statistics of the data set used in our experiments. To examine the generalization of models trained on tweets, the test data provided by the organizers consists of instances from different domains for both subtasks. Specifically, five corpora are included: LiveJournal(2014) is a collection of comments from LiveJournal blogs, SMS2013 is a SMS

\footnotetext{
${ }^{5}$ http://www.ark.cs.cmu.edu/TweetNLP/
} 
data set directly from last year, Twitter2013 is a twitter data set directly from last year, Twitter2014 is a new twitter data set and Twitter2014Sarcasm is a collection of tweets that contain sarcasm. Notice that the data set SMS2013 and Twitter2013 were also used as our development set. Form Table 1, we find that (1) the class distributions of test data sets almost agree with training data sets for both subtasks, (2) the percentages of class neutral in two subtasks are significantly different $(4.7 \%$ vs $45.5 \%$ ), which reflects that a sentence which is composed of different sentiment expressions may act neutrality, (3) Twitter2014Sarcasm data set is very small. According to the guideline, we did not use any development data for training in the evaluation period.

\begin{tabular}{lrrrr}
\hline data set & Positive & \multicolumn{1}{c}{ Negative } & \multicolumn{1}{c}{ Neutral } & Total \\
\hline subtask A: & & & & \\
train & $3,609(61 \%)$ & $2,023(34 \%)$ & $265(5 \%)$ & 5,897 \\
dev & $2,734(62 \%)$ & $1,541(35 \%)$ & $160(3 \%)$ & 4,435 \\
test & & & & \\
LiveJournal & $660(50 \%)$ & $511(39 \%)$ & $144(11 \%)$ & 1,315 \\
SMS2013 & $1,071(46 \%)$ & $1,104(47 \%)$ & $159(7 \%)$ & 2,334 \\
Twitter2013 & $2,734(62 \%)$ & $1,541(35 \%)$ & $160(3 \%)$ & 4,435 \\
Twitter2014 & $1,807(73 \%)$ & $578(23 \%)$ & $88(4 \%)$ & 2,473 \\
Twitter2014S & $82(66 \%)$ & $37(30 \%)$ & $5(4 \%)$ & 124 \\
all & $6,354(59 \%)$ & $3,771(35 \%)$ & $556(6 \%)$ & 10,681 \\
\hline subtask B: & & & & \\
train & $3,069(36 \%)$ & $1,313(15 \%)$ & $4,089(49 \%)$ & 8,471 \\
dev & $1,572(41 \%)$ & $601(16 \%)$ & $1,640(43 \%)$ & 3,813 \\
test & & & & \\
LiveJournal & $427(37 \%)$ & $304(27 \%)$ & $411(36 \%)$ & 1,142 \\
SMS2013 & $492(24 \%)$ & $394(19 \%)$ & $1,207(57 \%)$ & 2,093 \\
Twitter2013 & $1,572(41 \%)$ & $601(16 \%)$ & $1,640(43 \%)$ & 3,813 \\
Twitter2014 & $982(53 \%)$ & $202(11 \%)$ & $669(36 \%)$ & 1,853 \\
Twitter2014S & $33(38 \%)$ & $40(47 \%)$ & $13(15 \%)$ & 86 \\
all & $3,506(39 \%)$ & $1,541(17 \%)$ & $3,940(44 \%)$ & 8,987 \\
\hline
\end{tabular}

Table 1: Statistics of data sets in training (train), development (dev), test (test) set. Twitter2014S stands for Twitter2014Sarcasm.

We used macro-averaged F-measure of positive and negative classes (without neutral since it is margin in training data) to evaluate the performance of our systems and the averaged F-measure of five corpora was used to rank the final results.

\subsection{Submitted System Configurations}

For each subtask, each team can submit two runs: (1) constrained: only the provided data set can be used for training and no additional annotated data is allowed for training, however other resources such as lexicons are allowed; (2) unconstrained: any additional data can be used for training. We explored several classification algorithms on the development set and configured our final systems as follows. For constrained system, we used SVM and logistic regression algorithm implemented in scikit-learn toolkit (Pedregosa et al., 2011) to ad- dress two subtasks respectively and used selftraining strategy to conduct unconstrained system. Self-training is a semi-supervised learning method where a classifier is first trained with a small amount of labeled data and then we repeat the following procedure: the most confident predictions by the current classifier are added to training pool and then the classifier is retrained(Zhu, 2005). The parameters in constrained models and the growth size $k$ and iteration number $T$ in self-training are listed in Table 2 according to the results of preliminary experiments.

\begin{tabular}{lcc}
\hline task & constrained & unconstrained \\
\hline subtask A & SVM, kernel=rbf, c=500 & $k=100, T=40$ \\
subtask B & LogisticRegression, c=1 & $k=90, T=40$ \\
\hline
\end{tabular}

Table 2: System configurations for the constrained and unconstrained runs in two subtasks.

\section{Results and Discussion}

\subsection{Results}

We submitted four systems as described above and their final results are shown in Table 3, as well as the top-ranked systems released by the organizers. From the table, we observe the following findings.

Firstly, we find that the results of message-level polarity classification are much worse than the results of expression-level polarity disambiguation (82.93 vs 61.22) on both constrained and unconstrained systems, which is consistent with the previous work (Nakov et al., 2013). The low performance of message-level task may result from two possible reasons: (1) a message may contain mixed sentiments and (2) the strength of sentiments is different. In contrast, the texts in expression-level task are usually short and contain a single sentiment orientation, which leads to better performance.

Secondly, whether on constrained or unconstrained systems, the performance on Twitter2014Sarcasm data set is much worse than the performance on the other four data sets. This is because that sarcasm often expresses the opposite meaning of what it seems to say, that means the actual sentiment orientation of a word is opposite to its original orientation. Moreover, even for our human it is a challenge to identify whether it is a sarcasm or not.

Thirdly, the results on LiveJournal and SMS are comparable to the results on Twitter2013 and Twitter2014 in both subtasks, which indicates that 
online comments and SMS share some common characteristics with tweets (e.g., emoticons and punctuation). Therefore, in case of lack of labeled online comments or SMS data, we can use the existing tweets as training data instead.

Fourthly, our unconstrained systems exploit the test data of year 2014 in training stage and perform a worse result in subtask B. We speculate that the failure of using self-training on message-level data set is because that the performance of initial classifier was low and thus in the following iterations more and more noisy instances were selected to add the training pool, which eventually resulted in a final weak classifier.

In summary, we adopted some simple and basic features to classify the polarities of expressions and messages and they were promising. For subtask A, our systems rank 5th out of 19 submissions under the constrained setting and rank 2nd out of 6 submissions under the unconstrained setting. For subtask B, our systems rank 16th out of 42 submissions under the constrained setting and rank 5th out of 8 submissions under the unconstrained setting.

\subsection{Feature Combination Experiments}

To explore the effectiveness of different feature types, we conducted a series of feature combination experiments using the constrained setting as shown in Table 2 for both subtasks. For each time we repeated to add one feature type to current feature set and then selected the best one until all the feature types were processed. Table 4 shows the results of different feature combinations and the best results are shown in bold font.

From Table 4, we find that (1) MPQA, $n$-gram and Word_cluster are the most effective feature types to identify the polarities; (2) The POS tags make margin contribution to improve the performance since Stanford parser is designed for formal texts and in the future we may use specific parser instead; (3) The lexicon IMDB extracted from movie reviews has negative effects to classify twitter data, which indicates that there exist differences in the way of expressing sentiments between these two domains; (4) Twitter-specific features, i.e., hashtag and emoticon, are not as effective as expected. This is because they are sparse in the data sets. In subtask A with 16578 instances, only 292 instances (1.76\%) have hashtags and 419 instances $(2.52 \%)$ have emoticons. In subtask B with 17458 messages, more instances have hashtags (16.72\%) and emoticons (26.70\%). (5) For subtask A MPQA, $n$-gram, NRC and punctuation features achieve the best performance and for subtask B the best performance is achieved by using almost all features.

In summary, we find that $n$-gram and some lexicons such as MPQA are the most effective while twitter-specific features (i.e., hashtag and emoticon) are not as discriminating as expected and the main reason for this is that they are sparse in the data sets.

\begin{tabular}{ll|ll}
\hline Feature & \multicolumn{1}{c}{ Subtask A } & \multicolumn{1}{l}{ Feature } & \multicolumn{1}{l}{ Subtask B } \\
\hline MPQA & 77.49 & Word_cluster & 53.50 \\
.+n-gram & $80.08(2.59)$ & .+MPQA & $58.35(4.85)$ \\
.+NRC & $82.42(2.34)$ & .+W1Gram & $60.22(1.87)$ \\
.+Pun. & $\mathbf{8 3 . 8 3}(1.41)$ & .+Pun. & $60.99(0.77)$ \\
.+POS & $83.83(0)$ & .+Indicator & $61.38(0.39)$ \\
.+Emoticon & $83.49(-0.34)$ & .+SWN & $61.51(0.13)$ \\
.+Hashtag & $83.54(0.05)$ & .+Hashtag & $61.54(0.03)$ \\
.+IMDB & $83.51(-0.03)$ & .+n-gram & $61.56(0.02)$ \\
.+SWN & $82.92(-0.59)$ & .+Emoticon & $61.69(0.13)$ \\
- & - & .+POS & $\mathbf{6 1 . 7 1}(0.02)$ \\
- & - & .+IMDB & $61.11(-0.6)$ \\
- & - & .+NRC & $61.23(0.12)$ \\
\hline
\end{tabular}

Table 4: The results of feature combination experiments. The numbers in the brackets are the performance increments compared with the previous results. ".+" means to add current feature to the previous feature set.

\section{Conclusion}

In this paper we used several basic feature types to identify the sentiment polarity at expression level or message level and these feature types include $n$-gram, sentiment lexicon and twitter-specific features, etc. Although they are simple, our systems are still promising and rank above average (e.g., rank 5th out of 19 and 16th out of 42 in subtask A and $B$ respectively under the constrained setting). For the future work, we would like to analyze the distributions of different sentiments in sentences.

\section{Acknowledgments}

This research is supported by grants from $\mathrm{Na}-$ tional Natural Science Foundation of China (No.60903093) and Shanghai Knowledge Service Platform Project (No. ZF1213).

\section{References}

Apoorv Agarwal and Jasneet Sabharwal. 2012. Endto-end sentiment analysis of twitter data. In Pro- 


\begin{tabular}{|c|c|c|c|c|c|c|}
\hline Systems & LiveJournal & SMS2013 & Twitter2013 & Twitter2014 & Twitter2014S & Average \\
\hline A-constrained (expression-level) & 81.67 & 89.31 & 87.28 & 82.67 & 73.71 & 82.93 \\
\hline A-unconstrained & 81.69 & 89.26 & 87.29 & 82.93 & 73.71 & 82.98 \\
\hline NRC-Canada-A-constrained* & 85.49 & 88.03 & 90.14 & 86.63 & 77.13 & 85.48 \\
\hline Think_Positive-A-unconstrained ${ }^{*}$ & 80.90 & 87.65 & 88.06 & 82.05 & 76.74 & 83.08 \\
\hline B-constrained(message-level) & 69.44 & 59.75 & 62.31 & 63.17 & 51.43 & 61.22 \\
\hline B-unconstrained & 64.08 & 56.73 & 63.72 & 63.04 & 49.33 & 59.38 \\
\hline NRC-Canada-B-constrained* & 74.84 & 70.28 & 70.75 & 69.85 & 58.16 & 68.78 \\
\hline Think_Positive-B-unconstrained ${ }^{*}$ & 66.96 & 63.20 & 68.15 & 67.04 & 47.85 & 62.64 \\
\hline
\end{tabular}

Table 3: Performance of our systems and the top-ranked systems (marked with asterisk).

ceedings of the Workshop on Information Extraction and Entity Analytics on Social Media Data, pages 39-44, Mumbai, India, December. The COLING 2012 Organizing Committee.

Apoorv Agarwal, Boyi Xie, Ilia Vovsha, Owen Rambow, and Rebecca Passonneau. 2011. Sentiment analysis of twitter data. In Proceedings of the Workshop on Languages in Social Media, LSM '11, pages 30-38. Association for Computational Linguistics.

Stefano Baccianella, Andrea Esuli, and Fabrizio Sebastiani. 2010. Sentiwordnet 3.0: An enhanced lexical resource for sentiment analysis and opinion mining. In $L R E C$, volume 10, pages 2200-2204.

Luciano Barbosa and Junlan Feng. 2010. Robust sentiment detection on twitter from biased and noisy data. In Proceedings of the 23rd International Conference on Computational Linguistics: Posters, pages 36-44. Association for Computational Linguistics.

Lee Becker, George Erhart, David Skiba, and Valentine Matula. 2013. Avaya: Sentiment analysis on twitter with self-training and polarity lexicon expansion. In Proceedings of the Seventh International Workshop on Semantic Evaluation (SemEval 2013), pages 333-340. Association for Computational Linguistics, June.

Giacomo Berardi, Andrea Esuli, Diego Marcheggiani, and Fabrizio Sebastiani. 2011. Isti@ trec microblog track 2011: Exploring the use of hashtag segmentation and text quality ranking. In TREC.

Nadin Kökciyan, Arda Çelebi, Arzucan Özgür, and Suzan Üsküdarli. 2013. Bounce: Sentiment classification in twitter using rich feature sets. In Proceedings of the Seventh International Workshop on Semantic Evaluation (SemEval 2013), pages 554-561. Association for Computational Linguistics, June.

Andrew L Maas, Raymond E Daly, Peter T Pham, Dan Huang, Andrew Y Ng, and Christopher Potts. 2011. Learning word vectors for sentiment analysis. In Proceedings of the 49th Annual Meeting of the Association for Computational Linguistics: Human Language Technologies-Volume 1, pages 142-150. Association for Computational Linguistics.

Saif Mohammad, Svetlana Kiritchenko, and Xiaodan Zhu. 2013. Nrc-canada: Building the state-of-theart in sentiment analysis of tweets. In Proceedings of the Seventh International Workshop on Semantic Evaluation (SemEval 2013), pages 321-327. Association for Computational Linguistics, June.

Preslav Nakov, Sara Rosenthal, Zornitsa Kozareva, Veselin Stoyanov, Alan Ritter, and Theresa Wilson. 2013. Semeval-2013 task 2: Sentiment analysis in twitter. In Proceedings of the Seventh International Workshop on Semantic Evaluation (SemEval 2013), pages 312-320. Association for Computational Linguistics, June.

Alexander Pak and Patrick Paroubek. 2010. Twitter as a corpus for sentiment analysis and opinion mining. In Proceedings of the International Conference on Language Resources and Evaluation, LREC.

Bo Pang and Lillian Lee. 2008. Opinion mining and sentiment analysis. Foundations and trends in information retrieval, 2(1-2):1-135.

Fabian Pedregosa, Gaël. Varoquaux, Alexandre Gramfort, Vincent Michel, Bertrand Thirion, Olivier Grisel, Mathieu Blondel, Peter Prettenhofer, Ron Weiss, Vincent Dubourg, Jake Vanderplas, Alexandre Passos, David Cournapeau, Matthieu Brucher, Matthieu Perrot, and Édouard Duchesnay. 2011. Scikit-learn: Machine learning in Python. Journal of Machine Learning Research, 12:2825-2830.

Rosenthal Sara, Ritter Alan, Veselin Stoyanov, and Nakov Preslav. 2014. Semeval-2014 task 9: Sentiment analysis in twitter. In Proceedings of the Eighth International Workshop on Semantic Evaluation (SemEval'14). Association for Computational Linguistics, August.

Theresa Wilson, Janyce Wiebe, and Paul Hoffmann. 2009. Recognizing contextual polarity: An exploration of features for phrase-level sentiment analysis. Computational linguistics, pages 399-433.

Tian Tian Zhu, Fang Xi Zhang, and Man Lan. 2013. Ecnucs: A surface information based system description of sentiment analysis in twitter in the semeval-2013 (task 2). In Proceedings of the Seventh International Workshop on Semantic Evaluation (SemEval 2013), page 408.

Xiaojin Zhu. 2005. Semi-supervised learning literature survey. Technical Report 1530, Computer Sciences, University of Wisconsin-Madison. 